\title{
Effect of sea buckthorn meal extract in alleviating the toxic effect of ochratoxin $A$ and zearalenone in porcine peripheral blood mononuclear cells
}

\author{
Daniela Eliza Marin*1, Gina Cecilia Pistol ${ }^{1}$ \\ *Corresponding author: daniela.marin@ibna.ro
}

${ }^{1}$ National Research Development Institute for Animal Biology and Nutrition (IBNA), Calea Bucuresti No.1, Balotesti, 077015, Ilfov, Romania

\section{ABSTRACT}

The mycotoxins ochratoxin A (OTA) and zearalenone (ZEA) are frequent contaminants of cereals responsible for important toxic effects in human and animals. The aim of this study was to investigate the in vitro effect of the sea buckthorn meal extract in alleviating the toxic effect of ochratoxin A and zearalenone on oxidative stress and inflammation using porcine peripheral blood mononuclear cells. Our results have shown that both zearalenone and ochratoxin causes oxidative damage and alter the inflammatory response. The use of the sea buckthorn meal extract can improve some markers of the oxidative stress (total antioxidant status) and inflammation (proinflammatory cytokines IL-1 $\beta$, TNF- $\alpha$, IL-8, IL-6) altered by exposure to the mycotoxins ZEA and OTA and our results indicate that this waste represents a promising biological method that can be used for the alleviation of the mycotoxins negative effects.

Keywords: porcine blood mononuclear cells, swine, ochratoxin, zearalenone

INTRODUCTION

Mycotoxins are secondary metabolites produced mainly by fungus of Fusarium, Aspergillus, Penicillium, Alternaria and Claviceps genra, responsible for important toxic effects in animals and humans (Abrunhosa et al., 2016). Among them, ochratoxin A (OTA) and zearalenone (ZEA) are frequent contaminants of cereals (Lioi et al., 2004). Kidney is the main target of OTA, the exposure to the toxin lead to nephropathy in pigs and poultry (Stoev and Denev, 2013), while reproductive system is the main target of ZEA, the toxin having important estrogenic effects (Hueza et al., 2014). Both OTA and ZEA has also other toxic effects as genotoxic, hepatotoxic and immunotoxic (Sorrenti et al., 2013; Zinedine et al., 2007). 
Farm animals and in particular pigs are sensitive to mycotoxins due to the consumption of contaminated feed and important economic problems arise from the negative effect of the toxins on the animal health and reproduction capacity (Marin et al., 2013).

In comparation with physical and chemical methods for reducing the negative effects of mycotoxin, the use of the biological approaches is more recommended as they don't bound minerals or vitamins in the food/feed, are less toxic and could improve the health of the intoxicated animals through their rich content in bioactive substances (Varga et al., 2010). Recent studies have shown the efficacy of using agro-industrial wastes rich in bioactive compounds as counteracting solutions for alleviating the mycotoxins effect (Taranu et al., 2019).

Sea buckthorn (Hippophae rhamnoides) is a plant used in different fields as food, medicine and cosmetic industries (Ji et al., 2020a). The plant is rich in bioactive compounds as minerals, vitamins, unsaturated fatty acid, terpenoids, polyphenolic compounds, flavonoids, which have antioxidant and antiinflammatory effects (Zadernowski et al., 2005) .

The aim of this study was to investigate the in vitro effect of the sea buckthorn meal extract in alleviating the toxic effect of ochratoxin A and zearalenone on oxidative stress and inflammation using porcine peripheral blood mononuclear cells.

\section{MATERIALS AND METHODS}

Sea buckthorn meal extract. Sea buckthorn meal extract (SM) used in this study was obtained from S.C. OLEOMET S.R.L., Romania. The extraction of polyphenols from SM was performed as described by Pistol et al (Pistol et al., 2020) and Folin-Ciocalteu method was used for the assessment of the total polyphenol concentration expressed as $\mathrm{mg}$ of gallic acid equivalents (mg GAE)/100 g sea buckthorn meal.

Cell culture. Blood samples were obtained from jugular vein of commercially healthy piglets using tubes with anticoagulant (Vacutest, Italy). PBMC were isolated from blood as already described (Marin et al., 2011) and cultivated in complete medium: RPMI-1640 supplemented with $2 \mathrm{mM} \mathrm{L-}$ glutamine, $100 \mathrm{U} / \mathrm{mL}$ penicillin, $100 \mu \mathrm{g} / \mathrm{mL}$ streptomycin and $5 \%$ foetal calf serum (FCS) in cell culture plates. Cells were stimulated with $10 \mathrm{mg} / \mathrm{mL}$ LPS and treated with the following treatments $10 \mathrm{mM}$ toxin (OTA or ZEA) and/or sea buckthorn meal (SM) extract $(5 \mathrm{mg} / \mathrm{mL}$ gallic acid equivalents) for $48 \mathrm{~h}$ at $37^{\circ} \mathrm{C}$.

Cell viability assessment. MTT [3-(4,5-dimethylthiazol-2-yl)-2,5-diphenyl tetrazolium bromide] assay was used for assessing cell viability as already described (Marin et al., 2010). 
Determination of total antioxidant status. Total antioxidant capacity (TAC) assay was performed as already described (Marin et al., 2020) in cellular lysates and inhibition percentages were converted into trolox equivalent antioxidant capacity (TEAC), expressed as $\mu$ mol TEAC/g tissue.

Cytokine synthesis. ELISA kits (R\&D Systems, USA) were used for the quantification of the pro-inflammatory cytokine synthesis TNF- $\alpha$, IL-1 $\beta$, IL- 6 and IL-8 in the cell culture supernatants following the instruction of the manufacturer and as already described in our previous papers (Marin et al., 2019).

Statistical analysis. ANOVA tests were used to analyse the differences between treatments as compared to the control. The P values lower than 0.05 were considered significant.

\section{RESULTS AND DISCUSSION}

Effect of mycotoxins, sea buckthorn extract and their combination on PBMC viability. As it can be seen in the Figure 1, SM significantly increased the PBMC proliferation by $123 \%$. Treatment with $10 \mathrm{mM}$ ZEA or the combination of 10mM ZEA with SM extract has no significant effect on PBMC proliferation. By contrast, $10 \mathrm{mM}$ OTA induced a significant decrease of cell viability (by $38 \%$ ), while the concomitant administration of OTA and SM restore the PBMC proliferation near to the control. Previous studies have shown that ZEA has cytotoxic effect in PBMC isolated from different species (Morlett et al., 2015; Yang et al., 2016). In particular, zearalenone decrease the PBMC viability in swine (Marin et al., 2011; Yang et al., 2016) and it was shown that a concentration of $22.7 \mathrm{mM}$ of ZEA induce a decrease of PBMC viability with $50 \%$ (Marin et al, 2011). However, the concentration of ZEA used in our study $(10 \mathrm{mM})$ was too low to induce a significant decrease of PBMC viability.

\section{CELL PROLIFERATION}
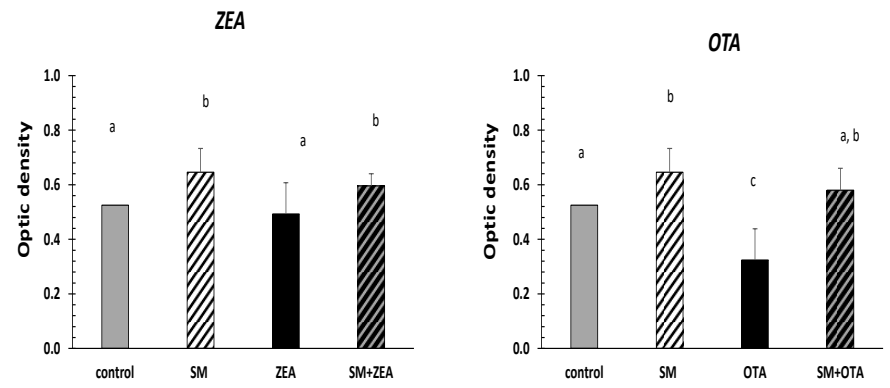

Figure 1. Effect of mycotoxins, sea buckthorn extract and their combination on PBMC viability 
Similarly, other studies have shown that OTA decreased cell viability of human PBMC (Liu et al., 2012; Periasamy et al., 2016; Stoev et al., 2009) and porcine PBMC (Mwanza et al., 2009), porcine PBMC being more sensitive to OTA toxicity as compared with human cells (Mwanza et al., 2009).

Administration of sea buckthorn extract improved OTA-induced cytotoxicity. Comparable results were obtained for other bioactive compounds, as resveratrol, that was able to improve cell proliferation decreased after ZEA exposure (Sang et al., 2016).

Effect of mycotoxins, sea buckthorn extract and their combination on antioxidant status. Both zearalenone and ochratoxin enhances ROS formation and causes oxidative damage (Qin et al., 2015; Tao et al., 2018).

\section{TOTAL ANTIOXIDANT CAPACITY}
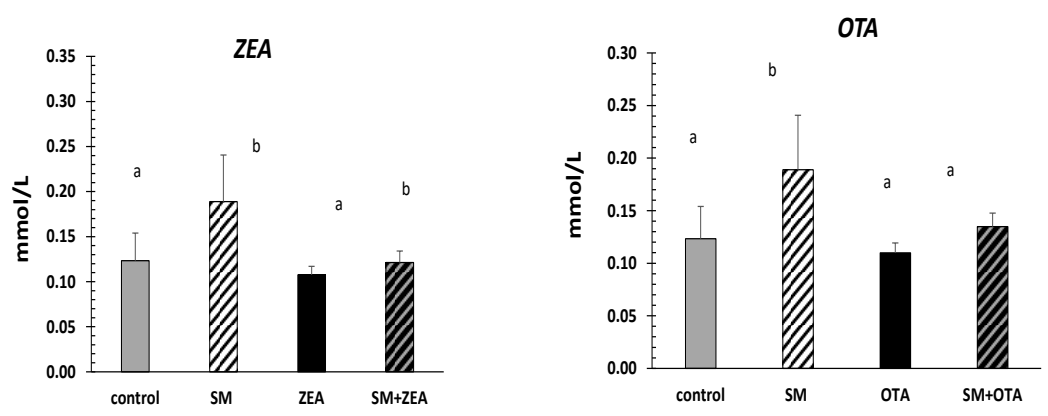

Figure 2. Effect of mycotoxins, sea buckthorn extract and their combination on oxidative status

However, in our experiment, neither ZEA or OTA were able to influence the total antioxidant capacity when they were administered to the cells in the presence or not of the SC extract (Figure 2). By contrast, SM extract alone significantly increase by 1.5 times the total antioxidant capacity (TAC) as compared with the control. Indeed, other biological wastes rich in bioactive compounds as grape seed meal waste increase the capacity of Caco 2 cells to respond to the oxidative stress (Nallathambi et al., 2020) and prevents oxidative DNA damage in rats PBMC (Aguiar et al., 2011)

Effect of mycotoxins, sea buckthorn extract and their combination on inflammation. Oxidative stress is correlated with inflammatory status as reactive oxygen species (ROS) play a major role in the inflammation progression (Mittal et al., 2014). Mycotoxins as well as bioactive extracts are known as modulators of different markers of inflammation. In our 
study, sea buckthorn meal extract reduced the concentration of proinflammatory cytokines TNF- $\alpha$, IL- 6 and IL-8 by $20.5 \%$; $87.4 \%$ and $45.2 \%$ respectively (Fig. 3).

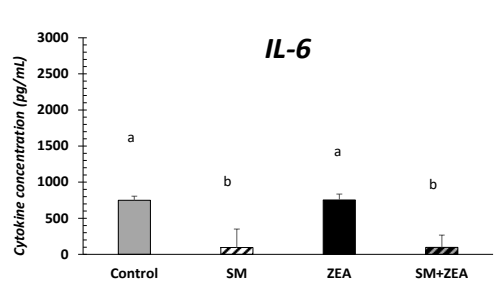

ZEA
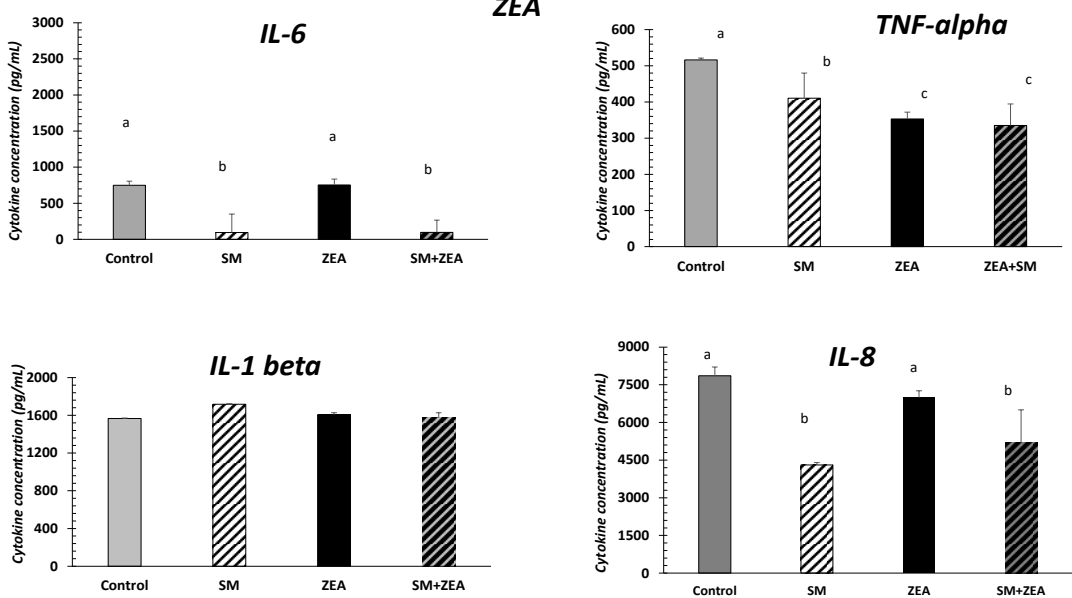

Figure 3. Effect of zearalenone, sea buckthorn extract and their combination on inflammation

OTA
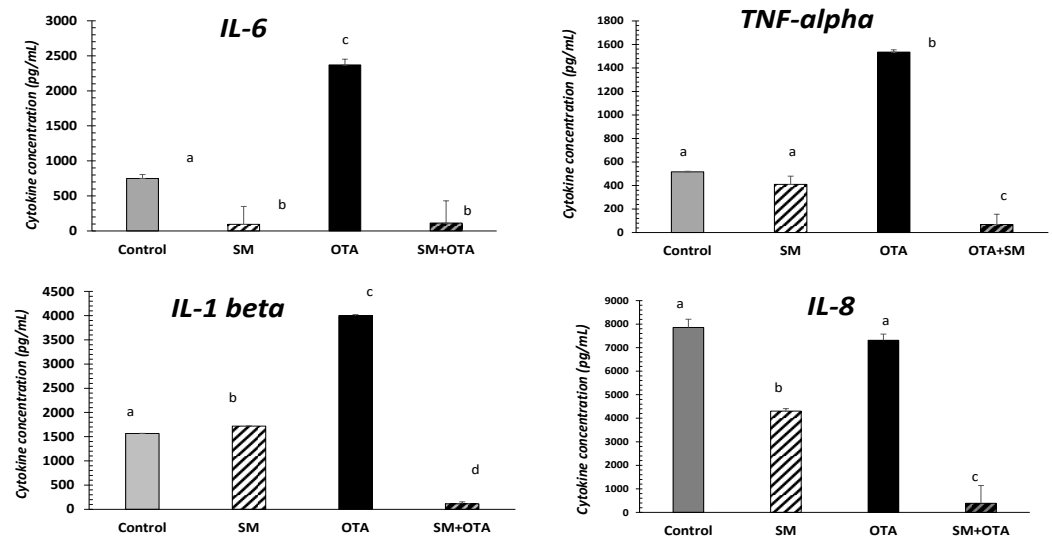

Figure 4. Effect of ochratoxin, sea buckthorn extract and their combination on inflammation

Our results are sustained by other studies showing that administration of sea buckthorn leaf extract in arthritis rat model, significantly reduced joint inflammation as compared to control (Ganju et al., 2005). By contrast, SM was 
not able to significantly decrease the concentration of IL-1b as compared with control.

The concentrations of the pro-inflammatory cytokines were not altered by the exposure of the PBMC to ZEA, with the exception of TNF-a concentration that was significantly decreased $(p<0.05)$ after $48 \mathrm{~h}$ exposure to the toxin. Literature data concerning inflammatory effect of ZEA are controversial as in vitro or in vivo studies have shown an increase or a decline of inflammatory response induced by ZEA depending on the cell type or organ (Bulgaru et al., 2021). In PBMC cells treated with both toxin and sea buckthorn meal extract, SM was able to decrease the synthesis of IL-6 (by 7.8 times) and IL-8 (by 1.3 times), but not of TNF- $\alpha$ and IL-1 $\beta$.

Previous studies have shown that OTA induces kidney, liver and intestinal toxicity, characterized by inflammation and cell death (Klahr and Morrissey, 2003). OTA has a more powerful inflammatory capacity than ZEA and significantly increase the synthesis of proinflammatory cytokines IL-6 (by 3.16 times), TNF- $\alpha$ (by 2.97 times), IL-1 $\beta$ (by 2.55 times) by PBMC, while having no effect on IL-8 synthesis ( $\mathrm{p}>0.05)$ - Fig. 4.

Concomitant exposure to both OTA and SM induced a high significant decrease $(p<0.0001)$ of pro-inflammatory cytokine synthesis IL-6, TNF- $\alpha$, IL$1 \beta$ and IL- 8 by $95 \%, 94 \%, 97 \%$ and $94 \%$, respectively, as compared with the OTA treatment.

\section{CONCLUSION}

In conclusion, our results have shown that the toxic effects of OTA were more important than that induced by ZEA on swine PBMC. Indeed, according to the UE recommendations concerning the guidance levels that can be accepted in feed for swine, the maximum accepted level for OTA is $50 \mathrm{ppm}$ while the level for ZEA is $100 \mathrm{ppm}$, that clearly reflect a higher toxicity of OTA as compared with ZEA. In this paper, we have demonstrated that the use of the sea buckthorn meal extract can improve some markers of the oxidative stress and inflammation altered by exposure to the mycotoxins ZEA and OTA. Sea buckthorn contains high concentrations of bioactive compounds as: vitamin C, carotenoids, tocopherols and polyphenols (flavonoids, phenolic acids and tannins) that are responsible for the antioxidant activity and antiinflammatory effect of the plant extract (Ji et al., 2020b) that are responsible for the SM beneficial activity observed in our study. This waste represents a promising biological method that can be used for the alleviation of the mycotoxins negative effects in farm animals. 


\section{ACKNOWLEDGEMENTS}

This work was financed thought the project ADER 9.2.1/2019 financed by Romanian Ministry of Agriculture.

\section{REFERENCES}

Abrunhosa, L., Morales, H., Soares, C., Calado, T., Vila-Chã, A.S., Pereira, M., Venâncio, A., 2016. A Review of Mycotoxins in Food and Feed Products in Portugal and Estimation of Probable Daily Intakes. Crit Rev Food Sci Nutr $56,249-265$.

Aguiar, O., Gollücke, A., Moraes, B., Pasquini, G., Catharino, R., Riccio, M., Ihara, S., Ribeiro, D., 2011. Grape juice concentrate prevents oxidative DNA damage in peripheral blood cells of rats subjected to a high-cholesterol diet. The British journal of nutrition 105, 694-702.

Bulgaru, C.V., Marin, D.E., Pistol, G.C., Taranu, I., 2021. Zearalenone and the Immune Response. Toxins (Basel) 13.

Ganju, L., Padwad, Y., Singh, R., Karan, D., Chanda, S., Chopra, M.K., Bhatnagar, P., Kashyap, R., Sawhney, R.C., 2005. Anti-inflammatory activity of Seabuckthorn (Hippophae rhamnoides) leaves. Int Immunopharmacol 5, 1675-1684.

Hueza, I.M., Raspantini, P.C.F., Raspantini, L.E.R., Latorre, A.O., Górniak, S.L., 2014. Zearalenone, an estrogenic mycotoxin, is an immunotoxic compound. Toxins (Basel) 6, 1080-1095.

Ji, M., Gong, X., Li, X., Wang, C., Li, M., 2020a. Advanced Research on the Antioxidant Activity and Mechanism of Polyphenols from Hippophae Species-A Review. Molecules (Basel, Switzerland) 25, 917.

Ji, M., Gong, X., Li, X., Wang, C., Li, M., 2020b. Advanced Research on the Antioxidant Activity and Mechanism of Polyphenols from Hippophae Species-A Review. Molecules 25, 917.

Klahr, S., Morrissey, J., 2003. Progression of chronic renal disease. Am J Kidney Dis 41, S3-7.

Lioi, M.B., Santoro, A., Barbieri, R., Salzano, S., Ursini, M.V., 2004. Ochratoxin A and zearalenone: a comparative study on genotoxic effects and cell death induced in bovine lymphocytes. Mutat Res 557, 19-27.

Liu, J., Wang, Y., Cui, J., Xing, L., Shen, H., Wu, S., Lian, H., Wang, J., Yan, X., Zhang, $X ., 2012$. Ochratoxin A induces oxidative DNA damage and G1 phase arrest in human peripheral blood mononuclear cells in vitro. Toxicol Lett 211, 164-171.

Marin, D.E., Bulgaru, C.V., Anghel, C.A., Pistol, G.C., Dore, M.I., Palade, M.L., Taranu, I., 2020. Grape Seed Waste Counteracts Aflatoxin B1 Toxicity in Piglet Mesenteric Lymph Nodes. Toxins (Basel) 12, 800.

Marin, D.E., Pistol, G.C., Bulgaru, C.V., Taranu, I., 2019. Cytotoxic and inflammatory effects of individual and combined exposure of HepG2 cells 
to zearalenone and its metabolites. Naunyn Schmiedebergs Arch Pharmacol 392, 937-947.

Marin, D.E., Pistol, G.C., Neagoe, I.V., Calin, L., Taranu, I., 2013. Effects of zearalenone on oxidative stress and inflammation in weanling piglets. Food Chem Toxicol 58, 408-415.

Marin, D.E., Taranu, I., Burlacu, R., Manda, G., Motiu, M., Neagoe, I., Dragomir, C., Stancu, M., Calin, L., 2011. Effects of zearalenone and its derivatives on porcine immune response. Toxicol In Vitro 25, 1981-1988.

Mittal, M., Siddiqui, M.R., Tran, K., Reddy, S.P., Malik, A.B., 2014. Reactive oxygen species in inflammation and tissue injury. Antioxidants \& redox signaling 20, 1126-1167.

Morlett, J., Viera-Limón, M., Sierra-Rivera, C., Luque Contreras, D., ZugastiCruz, A., 2015. Zearalenone Induced Cytotoxicity and Oxidative Stress in Human Peripheral Blood Leukocytes. Toxicol Open Access 1, 102.

Mwanza, M., Kametler, L., Bonai, A., Rajli, V., Kovacs, M., Dutton, M.F., 2009. The cytotoxic effect of fumonisin B1 and ochratoxin A on human and pig lymphocytes using the Methyl Thiazol Tetrazolium (MTT) assay. Mycotoxin Research 25, 233.

Nallathambi, R., Poulev, A., Zuk, J.B., Raskin, I., 2020. Proanthocyanidin-Rich Grape Seed Extract Reduces Inflammation and Oxidative Stress and Restores Tight Junction Barrier Function in Caco-2 Colon Cells. Nutrients $12,1623$.

Periasamy, R., Kalal, I.G., Krishnaswamy, R., Viswanadha, V., 2016. Quercetin protects human peripheral blood mononuclear cells from OTA-induced oxidative stress, genotoxicity, and inflammation. Environ Toxicol 31, 855-865.

Pistol, G.C., Marin, D.E., Rotar, M.C., Ropota, M., Taranu, I., 2020. Bioactive compounds from dietary whole grape seed meal improved colonic inflammation via inhibition of MAPKs and NF-kB signaling in pigs with DSS induced colitis. Journal of Functional Foods 66, 103708.

Qin, X., Cao, M., Lai, F., Yang, F., Ge, W., Zhang, X., Cheng, S., Sun, X., Qin, G., Shen, W., Li, L., 2015. Oxidative Stress Induced by Zearalenone in Porcine Granulosa Cells and Its Rescue by Curcumin In Vitro. PLOS ONE 10, e0127551.

Sang, Y., Li, W., Zhang, G., 2016. The protective effect of resveratrol against cytotoxicity induced by mycotoxin, zearalenone. Food \& Function 7, 3703-3715.

Sorrenti, V., Di Giacomo, C., Acquaviva, R., Barbagallo, I., Bognanno, M., Galvano, F., 2013. Toxicity of ochratoxin a and its modulation by antioxidants: a review. Toxins (Basel) 5, 1742-1766.

Stoev, S., Denev, S., Dutton, M., Nkosi, B., 2009. Cytotoxic Effect of Some Mycotoxins and their Combinations on Human Peripheral Blood Mononuclear Cells as Measured by the MTT Assay. The Open Toxinology 
Journal 2, 1-8.

Stoev, S.D., Denev, S.A., 2013. Porcine/chicken or human nephropathy as the result of joint mycotoxins interaction. Toxins (Basel) 5, 1503-1530.

Tao, Y., Xie, S., Xu, F., Liu, A., Wang, Y., Chen, D., Pan, Y., Huang, L., Peng, D., Wang, X., Yuan, Z., 2018. Ochratoxin A: Toxicity, oxidative stress and metabolism. Food Chem Toxicol 112, 320-331.

Taranu, I., Marin, D.E., Palade, M., Pistol, G.C., Chedea, V.S., Gras, M.A., Rotar, C., 2019. Assessment of the efficacy of a grape seed waste in counteracting the changes induced by aflatoxin B1 contaminated diet on performance, plasma, liver and intestinal tissues of pigs after weaning. Toxicon 162, 24-31.

Varga, J., Kocsubé, S., Péteri, Z., Vágvölgyi, C., Tóth, B., 2010. Chemical, physical and biological approaches to prevent ochratoxin induced toxicoses in humans and animals. Toxins (Basel) 2, 1718-1750.

Yang, L., Yang, W., Feng, Q., Huang, L., Zhang, G., Liu, F., Jiang, S., Yang, Z., 2016. Effects of purified zearalenone on selected immunological measurements of blood in post-weaning gilts. Animal Nutrition 2, 142-148.

Zadernowski, R., Naczk, M., Czaplicki, S., Rubinskiene, M., Szałkiewicz, M., 2005. Composition of phenolic acids in sea buckthorn (Hippophae rhamnoides L.) berries. Journal of the American Oil Chemists' Society 82, 175-179.

Zinedine, A., Soriano, J.M., Moltó, J.C., Mañes, J., 2007. Review on the toxicity, occurrence, metabolism, detoxification, regulations and intake of zearalenone: an oestrogenic mycotoxin. Food Chem Toxicol 45, 1-18. 